JOURNAL OF THE

AMERICAN MATHEMATICAL SOCIETY

Volume 14, Number 1, Pages 1-23

S 0894-0347(00)00352-0

Article electronically published on September 18, 2000

\title{
VALUES OF ZETA FUNCTIONS AT NEGATIVE INTEGERS, DEDEKIND SUMS AND TORIC GEOMETRY
}

\author{
STAVROS GAROUFALIDIS AND JAMES E. POMMERSHEIM \\ Dedicated to our teacher, W. Fulton
}

\section{INTRODUCTION}

In the present paper, we study relations among special values of zeta functions of real quadratic fields, properties of generalized Dedekind sums and Todd classes of toric varieties. The main theme of the paper is the use of toric geometry to explain in a conceptual way properties of the values of zeta functions and Dedekind sums, as well as to provide explicit computations.

Both toric varieties and zeta functions associate numerical invariants to cones in lattices, with different motivations and applications. Though we will focus on the case of two-dimensional cones in the present paper, we introduce notation and definitions that are valid for cones of arbitrary dimension. The reason for this added generality is clarity, as well as preparation for the results of a subsequent publication.

1.1. Zeta functions. We begin by reviewing the first source of numerical invariants of cones: the study of zeta functions.

Given a number field $K$, its zeta function is defined (for $\operatorname{Re}(s)$ sufficiently large) by:

$$
\zeta(K, s)=\sum_{\alpha} \frac{1}{Q(\alpha)^{s}}
$$

where the summation is over all nonzero ideals and $Q$ is the norm. The above function admits a meromorphic continuation in $\mathbb{C}$, with a simple pole at $s=1$, and regular everywhere else. Lichtenbaum [Li] conjectured a specific behavior of the zeta function $\zeta(K, s)$ at nonpositive integers related to the global arithmetic of the number field. In the special case of a totally real number field $K$, Lichtenbaum conjectured that the values of the zeta function at negative integers are rational numbers which involve the rank of the algebraic (or étale) $K$-theory of $K$. It is well understood that the zeta function of a totally real field $K$ can be decomposed as a

Received by the editors June 1, 1999 and, in revised form, May 24, 2000.

1991 Mathematics Subject Classification. Primary 11M06; Secondary 14M25, 11F20.

Key words and phrases. Zeta functions, Dedekind sums, toric varieties.

The authors were partially supported by NSF grants DMS-95-05105 and DMS-95-08972, respectively. 
sum $\zeta(K, s)=\sum_{\tau} \zeta_{Q, \tau}(s)$, where the sum is over a finite set of cones $\tau$ in lattices $M \subseteq K$ of $\operatorname{rank}[K: \mathbb{Q}]$ and

$$
\zeta_{Q, \tau}(s)=\sum_{a \in \tau \cap M-0} \frac{1}{Q(a)^{s}} .
$$

The reader may see [Sh1] and [Za2, Section 2] for a discussion.

The problem of calculating the zeta values $\zeta_{Q, \tau}(-n)$ for $n \geq 0$ for all triples $(M, Q, \tau)$ that arise from totally real fields has attracted a lot of attention by several authors. Klingen [Kl] and Siegel [Si1, Si2] (using analytic methods) proved that the values of the zeta functions of totally real fields at nonpositive integers are rational numbers and provided an algorithm for calculating them. Meanwhile, Shintani [Sh1 (using algebraic and combinatorial methods) gave an independent calculation of the zeta values $\zeta_{Q, \tau}(-n)$ for an arbitrary $Q$ which is a product of linear forms. Meyer $[\mathrm{Me}$ and Zagier Za3 gave another calculation of the zeta values $\zeta_{Q, \tau}(-n)$ for rank two lattices. Related results have also been obtained by P. Cassou-Noguès [CN1, CN2, CN3]. More recently, Hayes [Hs], Sczech [Sc1, Sc2] and Stevens $\left[\mathrm{St}\right.$ have constructed $\mathrm{PGL}_{m}(\mathbb{Q})$ cocycles which, among other things, provide a calculation of the zeta values (at nonpositive integers) of totally real number fields in terms of generalized Dedekind sums.

We now specialize to the case of real quadratic fields. We first note that when we use the word lattice, we will mean simply a free abelian group of finite rank. Some authors assume that a lattice comes equipped with a quadratic form, but we follow the usual custom in the theory of toric varieties, where no quadratic form is assumed to be present (for example, see $[\mathrm{Fu}]$ ). Given a lattice $M$, we will denote the associated real vector space $M \otimes \mathbb{R}$ by $M_{\mathbb{R}}$, and by a (rational) cone in $M$ or in $M_{\mathbb{R}}$, we will mean a cone generated by a finite set of rays from the origin which pass through points of $M$. From now on, we will use the following slightly normalized form of the zeta function $\zeta_{Q, \tau}$ defined for admissible triples $(M, Q, \tau)$ as follows:

Definition 1.1. An admissible triple $(M, Q, \tau)$ consists of a two-dimensional lattice $M$, a nonzero quadratic homogeneous function $Q: M_{\mathbb{R}} \rightarrow \mathbb{R}$ (i.e., a function satisfying $Q(a v)=a^{2} Q(v)$ for $a \in \mathbb{R}, v \in M_{\mathbb{R}}$ and such that $Q$ is not identically 0 ) and a rational two-dimensional cone $\tau$ in $M$ such that $Q$ is positive on $\tau$; that is, for all $a \in \tau, a \neq 0$, we have $Q(a)>0$. For an admissible triple $(M, Q, \tau)$ we set

$$
\zeta_{Q, \tau}(s)=\sum_{a \in \tau \cap M} \frac{w t(\tau, a)}{Q(a)^{s}}
$$

where $w t(\tau, \cdot): M \rightarrow \mathbb{Q}$ is the weight function defined by:

$$
w t(\tau, a)= \begin{cases}1 & \text { if } a \text { lies in the interior of } \tau, \\ 1 / 2 & \text { if } a \text { lies in the boundary of } \tau, \text { and } a \neq 0 \\ 0 & \text { otherwise }\end{cases}
$$

The above zeta function, defined for $\operatorname{Re}(s)$ sufficiently large, can be analytically continued to a meromorphic function on $\mathbb{C}$, regular everywhere except at 1 . Zagier [Za4] stated this in the case where $Q$ is indefinite, and the proof he gives works also in the definite case.

Note that all triples $(M, Q, \tau)$ that come from real quadratic fields are admissible. Zagier showed [Za4 that every triple $(M, Q, \tau)$ which arises from a real quadratic field can be constructed explicitly by means of a finite sequence $b=\left(b_{0}, \ldots, b_{r-1}\right)$ 
of integers greater than 1 and not all equal to 2. (It may be necessary to multiply $M$ by a totally positive number and $Q$ by a nonzero rational number, which simply multiplies values of the zeta function by a nonzero constant.) In addition, there are canonical vectors $A_{0}, \ldots, A_{r}$ in $M$ (that depend on $b$ ) that subdivide the cone $\tau=\left\langle A_{0}, A_{r}\right\rangle$ (i.e., the cone whose extreme rays are $A_{0}$ and $A_{r}$ ) in $M$ into $r$ nonsingular cones $\left\langle A_{i}, A_{i+1}\right\rangle$. For the convenience of the reader, as well as for motivation of the next theorem, we now recall Zagier's construction.

Given a sequence $b$ as above, we extend it to a sequence of integers parametrized by the integers by defining $b_{k}=b_{k \bmod r}$. Furthermore, for an integer $k$, we define

$$
w_{k}=\llbracket b_{k}, \ldots, b_{k+r-1} \rrbracket=b_{k}-\frac{1}{b_{k+1}-\frac{1}{b_{k+2}-\cdots}}
$$

where $\llbracket b_{k}, \ldots, b_{k+r-1} \rrbracket$ denotes the infinite periodic continued fraction with period $r$. Note that $w_{k}=w_{k+r}$ for all integers $k$, and that, by definition,

$$
w_{0}=b_{0}-\frac{1}{b_{1}-\frac{1}{\cdots b_{r-1}-\frac{1}{w_{0}}}}
$$

from which it follows that $w_{0}$ satisfies a quadratic equation $A w^{2}+B w+C=0$ where $A, B, C$ are (for a fixed $r$ ) polynomials in $b_{i}$ with integer coefficients. (Strictly speaking, $A, B, C$ are defined up to a scalar multiple; however, there is a canonical choice coming from writing the above continued fraction expansion as a quadratic equation.) Let $D=B^{2}-4 A C$ be the discriminant. Since $b_{i}>1$, it follows that $D>0$, and that the roots of the quadratic equation are $w_{0}=(-B+\sqrt{D}) /(2 A)$ and $w_{0}^{\prime}=(-B-\sqrt{D}) /(2 A)$ satisfying $w_{0}>1>w_{0}^{\prime}$. We define numbers $A_{0}=1$, $A_{k-1}=A_{k} w_{k}$ for $k \in \mathbb{Z}$. Since $w_{k}=b_{k}-\frac{1}{w_{k+1}}$, we see that

$$
A_{k-1}+A_{k+1}=b_{k} A_{k}
$$

for all integers $k$. Let us define $M=\mathbb{Z} w_{0}+\mathbb{Z}$ to be the rank two lattice in the real quadratic field $K=\mathbb{Q}(\sqrt{D})$ with basis $\left\{w_{0}, 1\right\}$. Since $A_{-1}=w_{0}, A_{0}=1$, the recursion relation (3) implies that $M=\mathbb{Z} A_{k}+\mathbb{Z} A_{k+1}$ for all integers $k$. In addition, the homogeneous function $Q: M_{\mathbb{R}} \rightarrow \mathbb{R}$ defined by

$$
Q\left(x w_{0}+y\right)=C x^{2}-B x y+A y^{2}
$$

is a multiple of the norm function of the real quadratic field $\mathbb{Q}(\sqrt{D})$, restricted to $M$; see also [Za4, p. 138].

We will now give an explicit formula for the zeta values $\zeta_{Q, \tau}(-n)$ (for $n \geq 0$ ), for the triples $(M, Q, \tau)$ constructed via a sequence $b$ above. While both the motivation and the proof involve concepts from the theory of toric geometry, the formula can be stated and understood without a knowledge of toric varieties. We will state the formula here, and in the next subsection we will discuss concepts from toric geometry which are necessary for the proof and which lead to a conceptual understanding of the present formula. 
Let $\lambda_{m}$ be defined by the power series:

$$
\frac{h}{1-e^{-h}}=\sum_{m=0}^{\infty} \lambda_{m} h^{m} ;
$$

thus we have: $\lambda_{m}=(-1)^{m} B_{m} / m$ ! where $B_{m}$ is the $m^{t h}$ Bernoulli number. (See also Definition 1.6 below.) Note that if $m>1$ is odd, then $\lambda_{m}=0$. For $n \geq 0$, define homogeneous polynomials $P_{n}(X, Y), R_{n}(X, Y)$ of degree $2 n$ by:

$$
\begin{aligned}
P_{n}(X, Y) & =\sum_{i+j=2 n, i, j \geq 0}(-1)^{i+1} \lambda_{i+1} \lambda_{j+1} X^{i} Y^{j}, \\
R_{n}(X, Y) & =\frac{X^{2 n+1}+Y^{2 n+1}}{X+Y}=X^{2 n}-X^{2 n-1} Y+\cdots+Y^{2 n} .
\end{aligned}
$$

We then have:

Theorem 1. For a sequence $b$, as above, with associated $(M, Q, \tau)$, the values $\zeta_{Q, \tau}(-n)$ for $n \geq 0$ are given explicitly as follows:

$$
\begin{aligned}
\zeta_{Q, \tau}(-n)= & P_{n}\left(\frac{\partial}{\partial x}, \frac{\partial}{\partial y}\right) \sum_{i=0}^{r-1}\left(Q\left(x A_{i-1}+y A_{i}\right)^{n}\right) \\
& +\lambda_{2 n+2} R_{n}\left(\frac{\partial}{\partial x}, \frac{\partial}{\partial y}\right) \sum_{i=0}^{r-1} b_{i}\left(Q\left(x A_{i-1}+y A_{i+1}\right)^{n}\right) .
\end{aligned}
$$

If the length $r$ of the sequence $b$ is fixed, the above expresses $\zeta_{Q, \tau}(-n)$ as a polynomial in the $b_{i}$ with rational coefficients, symmetric under cyclic permutation of the $b_{i}$.

In particular, we obtain the following formula due to Meyer; see also [Za1, Equation 3.3]:

$$
\zeta_{Q, \tau}(0)=\frac{1}{12} \sum_{i=0}^{r-1}\left(b_{i}-3\right) .
$$

It is important to note that in the above formula, not only do we see the cones $\left\langle A_{i-1}, A_{i}\right\rangle$ generated by consecutive rays, but also the cones $\left\langle A_{i-1}, A_{i+1}\right\rangle$ generated by rays two apart. In the classical formulas of Zagier, the cones generated by consecutive rays play a key role, but the other cones, those generated by rays two apart, seem to be an ingredient appearing for the first time in Theorem 1 above. This new ingredient permits one to express the zeta values in a somewhat simpler manner, as illustrated by the example below.

Example 1.2. We will express $\zeta_{Q, \tau}(-1)$ and $\zeta_{Q, \tau}(-2)$ using Theorem 11. For $i=$ $0, \ldots, r-1$, we define $L_{i}, M_{i}, N_{i}$ to be the coefficients of the quadratic form $Q$ on the $i^{\text {th }}$ nonsingular cone $\left\langle A_{i-1}, A_{i}\right\rangle$. Explicitly,

$$
Q\left(x A_{i-1}+y A_{i}\right)=L_{i} x^{2}+M_{i} x y+N_{i} y^{2} .
$$

We define $\tilde{L}_{i}, \tilde{M}_{i}, \tilde{N}_{i}$ similarly, as the coefficients of $Q$ on the cone $\left\langle A_{i-1}, A_{i+1}\right\rangle$, generated by rays two apart:

$$
Q\left(x A_{i-1}+y A_{i+1}\right)=\tilde{L}_{i} x^{2}+\tilde{M}_{i} x y+\tilde{N}_{i} y^{2} .
$$


Note that for sequences $b$ of fixed length $r, L_{i}, M_{i}, N_{i}, \tilde{L}_{i}, \tilde{M}_{i}, \tilde{N}_{i}$ are polynomials in $b_{i}$ with integer coefficients, as follows from Lemma 3.2. Theorem 1 then gives us:

$$
\zeta_{Q, \tau}(-1)=\frac{1}{720} \sum_{i=0}^{r-1}\left(5 M_{i}+b_{i}\left(-2 \tilde{L}_{i}+\tilde{M}_{i}-2 \tilde{N}_{i}\right)\right) .
$$

We may compare this with a formula of Zagier [Za4, p.149], which involves only the $L_{i}, M_{i}, N_{i}$ and not the $\tilde{L}_{i}, \tilde{M}_{i}, \tilde{N}_{i}$, though it does involve higher powers of the $b_{i}$ :

$$
\zeta_{Q, \tau}(-1)=\frac{1}{720} \sum_{i=0}^{r-1}\left(-2 N_{i} b_{i}^{3}+3 M_{i} b_{i}^{2}-6 L_{i} b_{i}+5 M_{i}\right) .
$$

The patient reader may use Lemma 3.2 to show that the above two expressions for $\zeta_{Q, \tau}$ are the same polynomial in the $b_{i}$ with rational coefficients.

As for $\zeta_{Q, \tau}(-2)$, Theorem 1 yields the following expression:

$$
\begin{aligned}
\zeta_{Q, \tau}(-2)=\frac{1}{15120} \sum_{i=0}^{r-1}( & -21 M_{i}\left(L_{i}+N_{i}\right) \\
& \left.+2 b_{i}\left(6 \tilde{L}_{i}^{2}-3 \tilde{L}_{i} \tilde{M}_{i}+2 \tilde{L}_{i} \tilde{N}_{i}+\tilde{M}_{i}^{2}-3 \tilde{M}_{i} \tilde{N}_{i}+6 \tilde{N}_{i}^{2}\right)\right) .
\end{aligned}
$$

1.2. Toric geometry. The second source of numerical invariants of cones comes from the theory of toric varieties. For a general reference on toric varieties, see [Da] or $[\mathrm{Fu}$.

Founded in the 1970s, the subject of toric varieties provides a strong link between algebraic geometry and the theory of convex bodies in a lattice. To each lattice polytope (the convex hull of a finite set of lattice points) is associated an algebraic variety with a natural torus action. This correspondence enables one to translate important properties and theorems about lattice polytopes into the language of algebraic geometry, and vice-versa. One important example of this is the very classical problem of counting the number of lattice points in a polytope. The early pioneers in the subject of toric varieties found that this problem, translated into algebraic geometry, becomes the problem of finding the Todd class of a toric variety. Much progress has been made over the past ten years on the Todd class problem, and this has led to a greater understanding of the lattice point counting question.

One approach to computing the Todd class of a toric variety is the fundamental work of R. Morelli [Mo. He settled a question of Danilov by proving a local formula expressing the Todd class of a toric variety as a cycle. Another approach, introduced by the second author in [P1, P2 , is to express the Todd class as a polynomial in the torus-invariant cycles. Dedekind sums appear as coefficients in these polynomials, and this leads to lattice point formulas in terms of Dedekind sums, as well as new reciprocity laws for Dedekind sums [P1]. Cappell and Shaneson [CS] subsequently announced an extension of the program of [P1] in which they proposed formulas for the Todd class of a toric variety in all dimensions. In [P3] it was shown that the polynomials of [P1, $\mathrm{P} 2]$ can be expressed nicely as the truncation of a certain power series whose coefficients were shown to be polynomial-time computable using an idea of Barvinok [Ba]. A beautiful power series expression for the equivariant Todd class of a toric variety was given by Brion and Vergne in [BV2]. Guillemin $\mathrm{Gu}$ also proved similar Todd class formulas from a symplectic geometry point of 
view. Furthermore, in [BV1], Brion and Vergne use the Todd power series of BV2] to give a formula for summing any polynomial function over a polytope.

The power series studied in [P3] and [BV1, BV2] are, in fact, identical (see Section (2) and play a central role in the present paper. A detailed discussion of the properties of these power series, which we call the Todd power series of a cone, is contained in Section 2. In this section, we state two theorems about the Todd power series of a two-dimensional cone that we will need in our study of the zeta function.

Given independent rays $\rho_{1}, \ldots, \rho_{n}$ from the origin in an $n$-dimensional lattice $N$, the convex hull of the rays in the vector space $N_{\mathbb{R}}=N \otimes \mathbb{R}$ forms an $n$-dimensional cone $\sigma=\left\langle\rho_{1}, \ldots, \rho_{n}\right\rangle$. Cones of this type (that is, ones generated by linearly independent rays) are called simplicial. Let $\mathcal{C}^{n}(N)$ denote the set of $n$-dimensional simplicial cones of $N$ with ordered rays. There is then a canonical function

$$
\mathfrak{t}: \mathcal{C}^{n}(N) \rightarrow \mathbb{Q} \llbracket x_{1}, \ldots, x_{n} \rrbracket,
$$

invariant under lattice automorphisms, which associates to each cone $\sigma$ a power series $\mathfrak{t}_{\sigma}$ with rational coefficients, called the Todd power series of $\sigma$. Several ways of characterizing this function are given in Section 2. These include an $N$-additivity property (see Proposition 2.1), an exponential sum over the cone (Proposition 2.3) and an explicit cyclotomic sum formula (Proposition 2.4).

In the case of a two-dimensional cone $\sigma$, the coefficient of $x y$ of $\mathfrak{t}_{\sigma}(x, y)$ was identified as a Dedekind sum [P1]. Furthermore, in [P1] it was shown that reciprocity formulas for Dedekind sums follow from an $N$-additivity formula for $\mathfrak{t}$. Zagier's higher-dimensional Dedekind sums [Za1] were later shown to appear as coefficients $\mathrm{BV} 2$. It is natural to conjecture that, in the two-dimensional case, all the coefficients of $\mathfrak{t}$ are generalized Dedekind sums and that reciprocity properties of generalized Dedekind sums will be related to the $N$-additivity formula of $\mathfrak{t}$. Indeed, this is the case; see Theorem 4 .

We now present an explicit link between Todd power series and zeta functions. The following theorem expresses the values of zeta functions at negative integers in terms of the Todd power series of a two-dimensional cone. Note that the idea of considering the Todd power series as a differential operator and applying this to an integral over a shifted cone is not new; this was introduced in $\mathrm{KP}$ and developed further in [BV1].

First we introduce some notation which is standard in the theory of toric varieties. If $\tau$ is a cone in a lattice $M$, the dual cone $\check{\tau}$ is a cone in the dual lattice $N=\operatorname{Hom}(M, \mathbb{Z})$, defined by

$$
\check{\tau}=\{v \in N \mid\langle v, u\rangle \geq 0 \text { for all } u \in \tau\} .
$$

The dual of an $n$-dimensional simplicial cone $\tau=\left\langle\rho_{1}, \ldots, \rho_{n}\right\rangle$ is generated by the rays $u_{i}$ normal to the $n-1$-dimensional faces of $\tau$; hence $\check{\tau}$ is also a simplicial cone. Given $h=\left(h_{1}, \ldots, h_{n}\right) \in \mathbb{R}^{n}$, we define the shifted cone $\tau(h)$ to be the following cone in $M_{\mathbb{R}}$ :

$$
\tau(h)=\left\{m \in M_{\mathbb{R}} \mid\left\langle u_{i}, m\right\rangle \geq-h_{i} \text { for all } i=1, \ldots, n\right\} .
$$

Here (and throughout) we have identified each ray $u_{i}$ with the primitive lattice point on that ray, that is, the nonzero lattice point on $u_{i}$ closest to the origin.

Given an $n$-dimensional simplicial cone $\tau=\left\langle\rho_{1}, \ldots, \rho_{n}\right\rangle$ in an $n$-dimensional lattice $M$, the multiplicity of $\tau$, denoted by $\operatorname{mult}(\tau)$, is defined to be the index in $M$ 
of the sublattice $\mathbb{Z} \rho_{1}+\cdots+\mathbb{Z} \rho_{n}$ (again identifying the rays $\rho_{i}$ with their primitive lattice points). Thus the multiplicity of $\tau$ is simply the volume of the parallelepiped formed by the vectors from the origin to the primitive lattice points on the rays of $\tau$.

Theorem 2. Let $\tau$ be a two-dimensional cone of multiplicity $q$ in a two-dimensional lattice $M$, and let $\sigma=\check{\tau}$ be the dual cone in $N=\operatorname{Hom}(M, \mathbb{Z})$. Then for all admissible triples $(M, Q, \tau)$ and $n \geq 0$, we have:

$$
\begin{aligned}
\zeta_{Q, \tau}(-n)= & (-1)^{n} n !\left\{\left(\mathfrak{t}_{\sigma}\right)_{(2 n+2)}\left(\frac{\partial}{\partial h_{1}}, \frac{\partial}{\partial h_{2}}\right)-\delta_{n, 0} \frac{q}{2} \frac{\partial^{2}}{\partial h_{1} \partial h_{2}}\right\} \\
& \diamond \int_{\tau(h)} \exp (-Q(u)) d u,
\end{aligned}
$$

where the diamond symbol above indicates that all derivatives are evaluated at $h_{1}=$ $h_{2}=0$ and $\delta_{n, 0}=1$ (resp. 0) if $n=0$ (resp. $\left.n \neq 0\right)$.

In this equation, $\left(\mathfrak{t}_{\sigma}\right)_{d}$ denotes the degree $d$ part of the Todd power series thought of as an (infinite order) constant coefficients differential operator acting on the function $h \rightarrow \int_{\tau(h)} \exp (-Q(u)) d u$.

Remark 1.3. We should point out that despite their similarity, Theorems 1 and 2 differ in their hypothesis, since there are admissible triples $(M, Q, \tau)$ that do not come from a sequence $b$.

The coefficients of $\mathfrak{t}_{\sigma}$ for a two-dimensional cone may be expressed explicitly in terms of continued fractions. We now state this formula. Let $\sigma$ be a twodimensional cone in a lattice $N$. Then there exists a (unique) pair of relatively prime integers $p, q$ with $0<p \leq q$ such that $\sigma$ is lattice equivalent (equivalent under the automorphism group of the lattice) to the cone $\sigma_{(p, q)} \stackrel{\text { def }}{=}\langle(1,0),(p, q)\rangle$ in $\mathbb{Z}^{2}$. Such a cone will be called a cone of type $(p, q)$. Let $b_{i}, h_{i}, k_{i}, X_{i}$ be defined in terms of the negative-regular continued fraction expansions:

$$
\frac{q}{p}=\left[b_{1}, \ldots, b_{r-1}\right], \quad \frac{h_{i}}{k_{i}} \stackrel{\text { def }}{=}\left[b_{1}, \ldots, b_{i-1}\right], \quad X_{i} \stackrel{\text { def }}{=}-h_{i} x+\left(q k_{i}-p h_{i}\right) y .
$$

(Throughout, we use such bracketed lists to denote finite negative-regular continued fractions.) We adopt the convention that $\left(h_{0}, k_{0}\right)=(0,-1)$ and $\left(h_{1}, k_{1}\right)=(1,0)$, so that $X_{0}=-q y$ and $X_{1}=-x-p y$.

We then have the following continued fraction expression for the degree $d$ part $\left(\mathfrak{t}_{\sigma}\right)_{d}$ of the Todd power series $\mathfrak{t}_{\sigma}$.

Theorem 3. For $\sigma$ of type $(p, q)$ as above, and for any integer $n \geq 0$, the degree $2 n+2$ part of the Todd power series $\mathfrak{t}_{\sigma}$ is expressed as follows:

$$
\begin{aligned}
\left(\mathfrak{t}_{\sigma}\right)_{2 n+2}(x, y)= & q x y \sum_{i=1}^{r} P_{n}\left(X_{i-1}, X_{i}\right)+\lambda_{2 n+2} q x y \sum_{i=1}^{r-1} b_{i} R_{n}\left(X_{i-1}, X_{i+1}\right) \\
& -\lambda_{2 n+2}\left(x X_{1}^{2 n+1}+y X_{r-1}^{2 n+1}\right)+\frac{1}{2} \delta_{n, 0} q x y .
\end{aligned}
$$

If $d \geq 1$ is odd, then $\left(\mathfrak{t}_{\sigma}\right)_{d}(x, y)=\frac{1}{2} \lambda_{d-1} q^{d-2} x y\left(x^{d-2}+y^{d-2}\right)$.

Remark 1.4. We have stated the formula above in the form we will need for our study of zeta functions. However, from the toric geometry point of view, it is more 
natural to use the continued fraction expansion of $\frac{q}{q-p}$ instead. This formula will be given in Section 2.3.

Remark 1.5. The formula for the Todd operator in Theorem 3 is reminiscent of formulas in quantum cohomology, even though we know of no conceptual explanation for this fact.

1.3. Dedekind sums. Finally, we calculate the coefficients of the Todd power series of two-dimensional cones in terms of a particular generalization $s_{i, j}$ (defined below) of the classical Dedekind sum. For an excellent review of the properties of the classical Dedekind sum, see [RG]. Several generalizations of the classical Dedekind sums were studied by T. Apostol, Carlitz, C. Meyer, D. Solomon, and more recently and generally by U. Halbritter $[\mathrm{AV}, \mathrm{Ha}, \mathrm{Me}, \mathrm{So}$. These papers investigate the sums (and several other generalizations of them, which we will not consider here) given in the following definition:

Definition 1.6. For relatively prime integers $p, q$ (with $q \neq 0)$, and for nonnegative integers $i, j$, we define the following generalized Dedekind sum:

$$
s_{i, j}(p, q)=\frac{1}{i ! j !} \sum_{a=1}^{q} \widehat{\operatorname{Ber}}_{i}\left(\left\langle\frac{a}{q}\right\rangle\right) \widehat{\operatorname{Ber}}_{j}\left(\left\langle-\frac{a p}{q}\right\rangle\right)
$$

where for a real number $x$, we denote by $\langle x\rangle$ the (unique) number such that $\langle x\rangle \in$ $(x+\mathbb{Z}) \cap(0,1]$. Here $\operatorname{Ber}_{m}$ denotes the $m^{t h}$ Bernoulli polynomial, defined by the power series $\sum_{m=0}^{\infty} \operatorname{Ber}_{m}(x) \frac{t^{m}}{m !}=\frac{t e^{t x}}{1-e^{t}}$, and $\widehat{\operatorname{Ber}}_{m}$ denotes the restriction of the $m^{\text {th }}$ Bernoulli polynomial $\mathrm{Ber}_{m}$ to $(0,1]$, with the boundary condition $\widehat{\operatorname{Ber}}_{m}(1) \stackrel{\text { def }}{=}$ $\frac{1}{2}\left(\operatorname{Ber}_{m}(1)+\operatorname{Ber}_{m}(0)\right)=B_{m}+\delta_{m, 1} / 2$, where $B_{m}$ is the $m^{t h}$ Bernoulli number, defined by $B_{m}=\operatorname{Ber}_{m}(0)$.1]

By definition, the sum $s_{1,1}(-p, q)$ coincides with the classical Dedekind sum $s(p, q)$ (cf. $[\mathrm{RG}$ ). An important property of generalized Dedekind sums is a reciprocity formula which leads to an evaluation in terms of negative-regular continued fractions. This reciprocity formula was most conveniently written by D. Solomon in terms of an additivity formula of a generating power series So Theorem 3.3]. On the other hand, the Todd operator also satisfies an additivity property. We denote by $f_{i, j}(p, q)$ (for nonnegative integers $i, j$ ) the coefficient of $x^{i} y^{j}$ of the power series $\mathfrak{t}_{\sigma_{(p, q)}}(x, y)$ (abbreviated by $\left.\mathfrak{t}_{(p, q)}(x, y)\right)$. We then have:

Theorem 4. Let $p, q \in \mathbb{Z}$ be relatively prime with $q \neq 0$. If $i, j>1$, then we have:

$$
f_{i, j}(p, q)=q^{i+j-1}(-1)^{i} s_{i, j}(p, q) .
$$

If $i=1$ or $j=1$, the above equation is true when the correction term

$$
q^{i+j-1}(-1)^{i+j} \frac{B_{i} B_{j}}{i ! j !}
$$

is added to the right hand side.

Corollary 1.7. Fixing $r$, for all nonnegative integers $i, j$ and with the notation of (8),$s_{i, j}(p, q)$ are polynomials in $b_{i}, 1 / q$.

\footnotetext{
${ }^{1}$ There seem to be two conventions for denoting Bernoulli numbers; one, which we follow here, is used often in number theory texts. Due to the facts that $B_{2 n+1}=0$ for $n \geq 1$, and that the sign of $B_{2 n}$ alternates, the other convention, which is often used in intersection theory, defines the $n^{\text {th }}$ Bernoulli number to be $(-1)^{n+1} B_{2 n}$.
} 
Remark 1.8. For $i=j=1$, the above theorem was obtained in [P1], and was a motivation for the results of the present paper.

1.4. Is toric geometry needed? Some natural questions arise, at this point:

- Is toric geometry needed?

- How do the statement and proof of Theorem 1 differ from the statement and proof of Zagier's formula [Za4]?

With respect to the first question, Theorem 1 (an evaluation of zeta functions associated to real quadratic fields) is stated without reference to toric geometry, and a close examination reveals that its proof is based on an analytic Lemma 2.11 and on the two-dimensional analogue of the Euler-MacLaurin formula given by Proposition 2.13. In addition, two-dimensional cones can be canonically subdivided into nonsingular cones, so in a sense the two-dimensional analogue of the EulerMacLaurin formula can be obtained by the classical (one-dimensional) one, as is used by Zagier [Za4]. Furthermore, number theory offers, for every $m \geq 2$, a canonical Eisenstein cocycle of $\mathrm{PGL}_{m}(\mathbb{Q})[\mathrm{Sc1}, \mathrm{Sc2}]$ that expresses, among other things, the generalized Dedekind sums $s_{i, j}$ in terms of negative-regular continued fraction expansions like the ones of Theorems 3 and 4 , and the values (at nonpositive integers) of zeta functions of totally real fields (of degree $m$ ) in terms of generalized Dedekind sums. See also [So, $\underline{\mathrm{St}}]$.

On the other hand, for every simplicial cone $\sigma$ in an $m$-dimensional lattice $N$, toric geometry constructs a canonical Todd power series $\mathfrak{t}_{\sigma}$ satisfying an additivity property (see Proposition 2.1 below). The coefficients of the Todd power series are generalized Dedekind sums, and the power series itself is intimately related to the Euler-MacLaurin summation formula. As a result, for $m=2$, we provide a toric geometry explanation of Theorems 1 and 4 .

With respect to the second question, Zagier [Za4] obtained similar formulas for the values of the zeta function of a quadratic number field at negative integers. Zagier used additivity in the lattice $M$, whereas we use additivity in the dual lattice $N$. The definition of the zeta function of an arbitrary rational cone $\tau$ involves a sum over the lattice points in that cone, which sits inside a two-dimensional lattice $M$. A major idea in Zagier's attack on finding values of these zeta functions was to subdivide the cone $\tau$ into nonsingular cones, and use the set-theoretic additivity of summations under subdivisions. Precisely, the function $\zeta_{Q},(s): \mathcal{C}^{2}(M) \rightarrow \mathbb{C}$ is additive, i.e., it satisfies:

$$
\zeta_{Q, \tau}(s)=\zeta_{Q, \tau_{1}}(s)+\zeta_{Q, \tau_{2}}(s) .
$$

This kind of additivity may be called $M$-additivity, since it is nothing more than set-theoretic additivity in the lattice $M$. Note that the $M$-additivity of $\zeta_{Q}$,. $(s)$ is due to the particular choice of the weight function $w t$ involved in the definition.

The approach of this paper is to use a different and somewhat more subtle kind of additivity in the dual lattice, which may be called $N$-additivity. This idea may be illustrated by considering the function $g$ that sends a rational cone $\tau$ in a lattice $M \equiv \mathbb{Z}^{n}$ to the sum

$$
g(\tau)=\sum_{n \in \check{\tau} \cap N} x^{n}
$$


which defines a rational function. The function $g$ is then additive in the sense that if an $n$-dimensional cone $\tau$ is subdivided into $n$-dimensional cones $\tau_{i}$, then

$$
g(\tau)=\sum_{i} g\left(\tau_{i}\right) .
$$

This additivity arises in the work of Brion $([\mathrm{Br})$, and is also discussed in $\mathrm{P} 3$ and $[\mathrm{BP}]$. It is important to realize that in this $N$-additivity formula, cones of smaller dimension may be completely ignored, whereas these lower-dimensional cones must be accounted for in the $M$-additivity, which is inclusion-exclusion. For the present work, we rely on the $N$-additivity of the Todd operator, i.e., the function sending a cone $\sigma$ to the power series $\mathfrak{t}_{\sigma}$, which is $N$-additive with a suitable change of coordinates (see Proposition 2.1). These somewhat deeper ideas prove to be effective in the study of zeta functions, for they imply, as we show in this paper, that the zeta values are a priori polynomials in $b_{i}$ and $1 / q$; moreover, these polynomials are directly defined in terms of the Todd operator and the quadratic form $Q$.

1.5. Plan of the proof. In Section 2, we review well-known properties of Todd power series and prove Theorems 2 and 3 . In Section 3 we review the relation between the zeta functions that we consider and the zeta functions of real quadratic fields. We give a detailed construction of zeta functions, and prove Theorem 1 Finally, in Section 4 we review properties of generalized Dedekind sums and prove Theorem 4

Acknowledgments. We wish to thank M. Rosen for encouraging conversations during the academic year 1995-96. We also thank B. Sturmfels and M. Brion for their guidance. We especially wish to thank W. Fulton for enlightening and encouraging conversations since our early years of graduate studies.

\section{The TODD POWER SERIES OF A CONE}

In this section we study properties of the Todd power series $\mathfrak{t}_{\sigma}$ associated to a simplicial cone $\sigma$. Section 2.1 provides an introduction and statements of several previously discovered formulas for the Todd power series. In Section 2.2 we make these formulas explicit for two-dimensional cones. Section 2.3 contains a proof of the explicit continued fraction formula for the Todd power series of a two-dimensional cone. Finally, in Section 2.4 we prove Theorem 2 which links Todd power series with the problem of evaluating zeta functions and nonpositive integers.

2.1. General properties of the Todd power series. Todd power series were studied in connection with the Todd class of a simplicial toric variety in [P3]. Independently, they were introduced in BV2] in the study of the equivariant Todd class of a simplicial toric variety. In addition, these power series appear in Brion and Vergne's formula for counting lattice points in a simple polytope [BV1], which is an extension of Khovanskii and Pukhlikov's formula [KP]. In these remarkable formulas, the power series in question are considered as differential operators which are applied to the volume of a deformed polytope. The result yields the number of lattice points in the polytope, or more generally, the sum of any polynomial function over the lattice points in the polytope. Below (Proposition 2.13), we give a version of this formula expressing the sum of certain functions over the lattice points contained in a simplicial cone. 
The Todd power series considered in the works cited above are also closely related to the fundamental work of $\mathrm{R}$. Morelli on the Todd class of a toric variety [Mo]. A precise connection is given in [P3, Section 1.8].

We now state some of the properties of the Todd power series of a simplicial cone. Our purpose is twofold: we will need these properties in our application to zeta functions, and we wish to unite the approaches of the works cited above. Here, we follow the notation of $\mathrm{P} 3$.

Let $\sigma=\left\langle\rho_{1}, \ldots, \rho_{n}\right\rangle$ be an $n$-dimensional simplicial cone in an $n$-dimensional lattice $N$. The Todd power series $\mathfrak{t}_{\sigma}$ of $\sigma$ is a power series with rational coefficients in variables $x_{1}, \ldots, x_{n}$ corresponding to the rays of $\sigma$. These power series, when evaluated at certain divisor classes, yield the Todd class of any simplicial toric variety [P3, Theorem 1].

To state the properties of $\mathfrak{t}_{\sigma}$, it will be useful to consider the following variant $\mathfrak{s}$ of the power series $\mathfrak{t}$ defined in P3] by:

$$
\mathfrak{s}_{\sigma}\left(x_{1}, \ldots, x_{n}\right)=\frac{1}{\operatorname{mult}(\sigma) x_{1} \cdots x_{n}} \mathfrak{t}_{\sigma}\left(x_{1}, \ldots, x_{n}\right),
$$

which is a Laurent series in $x_{1}, \ldots, x_{n}$.

The Todd power series $\mathfrak{t}_{\sigma}$ and $\mathfrak{s}_{\sigma}$ are characterized by the following proposition [P3. Theorem 2], which states that $\mathfrak{s}$ is additive under subdivisions (after suitable coordinate changes), and gives the value of $\mathfrak{s}$ on nonsingular cones. An $n$-dimensional cone is called nonsingular if it is generated by rays forming a basis of the lattice. It is well known that any cone may be subdivided into nonsingular cones, and that such a subdivision determines a resolution of singularities of the corresponding toric variety (cf. [Fu, Section 2.6]).

Proposition 2.1. If $\Gamma$ is a simplicial subdivision of $\sigma$, then

$$
\mathfrak{s}_{\sigma}(X)=\sum_{\gamma \in \Gamma_{(n)}} \mathfrak{s}_{\gamma}\left(\gamma^{-1} \sigma X\right)
$$

where the sum is taken over the $n$-dimensional cones of the subdivision $\Gamma$. Here $X$ denotes the column vector $\left(x_{1}, \ldots, x_{n}\right)^{t}$ and we have identified each cone ( $\sigma$ and $\gamma$ ) with the $n$-by-n matrix whose columns are the coordinates of the rays of that cone.

For nonsingular cones, $\sigma$, we have the following expression for $\mathfrak{s}_{\sigma}$ :

$$
\mathfrak{s}_{\sigma}\left(x_{1}, \ldots, x_{n}\right)=\prod_{i=1}^{n} \frac{1}{1-e^{-x_{i}}} .
$$

Remark 2.2. It follows immediately that for any cone $\sigma, \mathfrak{s}_{\sigma}$ is a rational function of the $e^{x_{i}}$.

The Laurent series $\mathfrak{s}_{\sigma}$ in $x_{1}, \ldots, x_{n}$ may also be expressed as an exponential sum over the lattice points in the cone, in the spirit of the important earlier work of M. Brion $[\mathrm{Br}$.

Proposition 2.3. Let $\check{\sigma}$ denote the dual cone in the lattice $M=\operatorname{Hom}(N, \mathbb{Z})$. We then have

$$
\mathfrak{s}_{\sigma}\left(x_{1}, \ldots, x_{n}\right)=\sum_{m \in \sigma \circ \cap M} e^{-\left(\left\langle m, \rho_{1}\right\rangle x_{1}+\cdots+\left\langle m, \rho_{n}\right\rangle x_{n}\right)} .
$$

The equality is one of rational functions in the $e^{x_{i}}$. 
Proof. As noted in $[\mathrm{Br}]$, since $\left\langle m, \rho_{i}\right\rangle \geq 0$ for $m \in \check{\sigma} \cap M$, the right hand side has a meaning in the completion of $\mathbb{C}\left[y_{1}, \ldots, y_{n}\right]$ with respect to the ideal $\left(y_{1}, \ldots, y_{n}\right)$, where $y_{i}$ stands for $e^{-x_{i}}$. While it is not obvious, the right hand side is a rational function of the $e^{x_{i}}$. See [Br, p.654]. The left hand side is a rational function of the $e^{x_{i}}$ by Proposition 2.1. By [Br p.655] and the second formula of Proposition 2.1 these two rational functions are equal on nonsingular cones. As any cone can be subdivided into nonsingular cones, it suffices to verify that the right hand side satisfies the additivity formula of Proposition 2.1. But this follows again from Brion's work. See the proposition of [Br] p.657], for example. Intuitively, this additivity can be seen from the fact that a sum of exponentials over a cone containing a straight line vanishes formally.

The $\mathfrak{s}_{\sigma}$ also have an explicit expression in terms of cyclotomic sums, due to Brion and Vergne. Following [BV2], we introduce the following notation. Let $u_{1}, \ldots, u_{n}$ denote the primitive generators of the dual cone $\check{\sigma}$. Thus we have $\left\langle u_{i}, \rho_{j}\right\rangle=0$ if $i \neq j$, and $\left\langle u_{i}, \rho_{i}\right\rangle \in \mathbb{Z}$, but does not necessarily equal 1 . Let $N_{\sigma}$ be the subgroup of $N$ generated by the $\rho_{i}, i=1, \ldots, n$, and let $G_{\sigma}=N / N_{\sigma}$. Then $G_{\sigma}$ is an abelian group of order mult $(\sigma)$, which we denote by $q$. Define characters $a_{i}$ of $G_{\sigma}$ by

$$
a_{i}(g)=e^{2 \pi i \frac{\left\langle u_{i}, g\right\rangle}{\left\langle u_{i}, \rho_{i}\right\rangle}} .
$$

Proposition 2.4. The Laurent series $\mathfrak{s}_{\sigma}$ coincides with Brion and Vergne's formula expressing their Todd differential operator. Namely, we have

$$
\mathfrak{s}_{\sigma}\left(x_{1}, \ldots, x_{n}\right)=\frac{1}{q} \sum_{g \in G_{\sigma}} \prod_{i=1}^{n} \frac{1}{1-a_{i}(g) e^{-x_{i}}} .
$$

Proof. By inspection, the right hand side is a rational function in the variables $y_{i}=$ $e^{-x_{i}}$ which takes the value 1 when $y_{i}=0$ for all $i$. Such rational functions embed into the completion of the ring $\mathbb{C}\left[y_{1}, \ldots, y_{n}\right]$ with respect to the ideal $\left(y_{1}, \ldots, y_{n}\right)$. To prove the proposition, it is enough to show that the right hand side and the right hand side of Proposition 2.3 define the same element of this completion. To do so, we expand the right hand side, getting:

$$
\frac{1}{q} \sum_{k_{1}, \ldots, k_{n} \geq 0} \sum_{g \in G_{\sigma}} \prod_{i=1}^{n}\left[a_{i}(g) e^{-x_{i}}\right]^{k_{i}}
$$

which becomes

$$
\frac{1}{q} \sum_{k_{1}, \ldots, k_{n} \geq 0} e^{-\left(k_{1} x_{1}+\cdots+k_{n} x_{n}\right)} \sum_{g \in G_{\sigma}} a_{1}^{k_{1}} \cdots a_{n}^{k_{n}}(g)
$$

This last sum over $G_{\sigma}$ is either $q$ or 0 , depending on whether $a_{1}^{k_{1}} \cdots a_{n}^{k_{n}}$ is the trivial character of $G_{\sigma}$ or not. Comparing the above with the right hand side of Proposition 2, we see that it suffices to show that $a_{1}^{k_{1}} \cdots a_{n}^{k_{n}}$ is the trivial character of $G_{\sigma}$ if and only if there exists $m \in \check{\sigma}$ such that $\left\langle m, \rho_{i}\right\rangle=k_{i}$ for all $i$. This straightforward lattice calculation is omitted.

Remark 2.5. The sum in the proposition above appears in the important work of R. Diaz and S. Robins $[\overline{D R}$. They use such sums to give an explicit formula for the number of lattice points in a simple polytope. Interestingly, their techniques, which come from Fourier analysis, are seemingly unrelated to the toric geometry discussed above. 
2.2. Properties of Todd power series of two-dimensional cones. In this section, we state properties of the power series $\mathfrak{t}_{\sigma}$ for a two-dimensional cone $\sigma$.

It is not hard to see that if $\sigma$ is any two-dimensional cone, then there are relatively prime integers $p, q$ such that $\sigma$ is lattice-equivalent to the cone

$$
\sigma_{(p, q)} \stackrel{\text { def }}{=}\langle(1,0),(p, q)\rangle \subset \mathbb{Z}^{2} .
$$

Here $q$ is determined up to sign and $p$ is determined modulo $q$. Thus we may arrange to have $q>0$ and $0 \leq p<q$. This gives a complete classification of twodimensional cones up to lattice isomorphism. In discussing Todd power series we will abbreviate $\mathfrak{t}_{\sigma_{(p, q)}}$ by $\mathfrak{t}_{(p, q)}$.

The explicit cyclotomic formula of Proposition 2.4 may be written as:

Proposition 2.6. The Todd power series of a two-dimensional cone is given by

$$
\mathfrak{t}_{(p, q)}(x, y)=\sum_{\omega^{q}=1} \frac{x y}{\left(1-\omega^{-p} e^{-x}\right)\left(1-\omega e^{-y}\right)} .
$$

Proof. With the coordinates above and the notation of Proposition 2.4, we have $u_{1}=(q,-p), u_{2}=(0,1)$, and $G_{\sigma}$ consists of the lattice points $(0, k), k=0, \ldots, q-1$. The desired equation now follows directly from Proposition 2.4.

In the two-dimensional case, the additivity formula of Proposition 2.1 can be expressed as an explicit reciprocity law. This and a periodicity relation for $\mathfrak{s}$ are contained in the following theorem.

Proposition 2.7. Let $p$ and $q$ be relatively prime positive integers. Then

$$
\begin{aligned}
\mathfrak{s}_{(p, q)}\left(x-\frac{p}{q} y, \frac{1}{q} y\right)+\mathfrak{s}_{(q, p)}\left(y-\frac{q}{p} x, \frac{1}{p} x\right) & =\mathfrak{s}_{(0,1)}(x, y), \\
\mathfrak{s}_{(p+q, q)}(x, y) & =\mathfrak{s}_{(p, q)}(x, y) .
\end{aligned}
$$

Proof. The quadrant $\langle(1,0),(0,1)\rangle$ may be subdivided into cones $\gamma_{1}=\langle(1,0),(p, q)\rangle$ and $\gamma_{2}=\langle(0,1),(p, q)\rangle$. The cone $\gamma_{1}$ is of type $(p, q)$, and $\gamma_{2}$ is of type $(q, p)$. Applying the additivity formula (Proposition [2.1) to this subdivision yields the first equation above.

The second equation follows from the fact that the cones $\langle(1,0),(p, q)\rangle$ and $\langle(1,0),(p+q, q)\rangle$ are lattice isomorphic.

Let $\mathfrak{s}^{e v}\left(\right.$ resp. $\left.\mathfrak{s}^{\text {odd }}\right)$ denote the part of $\mathfrak{s}$ of even (resp. odd) total degree.

Corollary 2.8. Given a two-dimensional lattice $N$, the function $\mathfrak{s}: \mathcal{C}^{2}(N) \rightarrow$ $\mathbb{Q}((x, y))$ (where $\mathbb{Q}((x, y))$ is the function field of the power series ring $\mathbb{Q} \llbracket x, y \rrbracket)$ is uniquely determined by properties (13) and (14) and its initial condition $\mathfrak{s}_{(0,1)}(x, y)$ $=1 /\left(\left(1-e^{-x}\right)\left(1-e^{-y}\right)\right)$. Since equations (13) and (14) are homogeneous with respect to the degrees of $x$ and $y$, it follows that $\mathfrak{s}^{\text {ev }}$ (resp. $\mathfrak{s}^{\text {odd }}$ ) satisfies (13) and (14) with initial condition $\mathfrak{s}_{(0,1)}^{\text {ev }}\left(\right.$ resp. $\left.\mathfrak{s}_{(0,1)}^{\text {odd }}\right)$.

Remark 2.9. Corollary 2.8 has a converse. From equations (13) and (14) it follows that $\mathfrak{s}_{(0,1)}$ satisfies the following relations:

$$
\mathfrak{s}_{(0,1)}(x, y)=\mathfrak{s}_{(0,1)}(x-y, y)+\mathfrak{s}_{(0,1)}(y-x, x) \quad \text { and } \quad \mathfrak{s}_{(0,1)}(x, y)=\mathfrak{s}_{(0,1)}(y, x) .
$$


Conversely, one can show that, given any element $g(x, y) \in \mathbb{Q}((x, y))$ satisfying

$$
g(x, y)=g(x-y, y)+g(y-x, x) \text { and } g(x, y)=g(y, x)
$$

there is a unique function $\mathfrak{g}: \mathcal{C}^{2}(N) \rightarrow \mathbb{Q}((x, y))$ so that $\mathfrak{g}_{(0,1)}=g$.

2.3. Continued fraction expansion for the Todd power series. In this section, we prove Theorem 3 which expresses the coefficients in the Todd power series of a two-dimensional cone in terms of continued fractions. Before doing so, we first formulate an equivalent version which is more natural from the point of view of toric varieties. The continued fraction expansion in this second version of the formula corresponds directly to a desingularization of the cone.

Given relatively prime integers $p, q$ with $p>0$ and $0 \leq p<q$, let $a_{i}, \gamma_{i}, \delta_{i}, L_{i}$ be defined in terms of the negative continued fraction expansions:

$\frac{q}{q-p}=\left[a_{1}, \ldots, a_{s-1}\right], \quad \frac{\gamma_{i}}{\delta_{i}} \stackrel{\text { def }}{=}\left[a_{1}, \ldots, a_{i-1}\right], \quad L_{i} \stackrel{\text { def }}{=} \gamma_{i} x+\left(q \delta_{i}+(p-q) \gamma_{i}\right) y$,

with the convention that $\left(\gamma_{0}, \delta_{0}\right)=(0,-1)$ and $\left(\gamma_{1}, \delta_{1}\right)=(1,0)$, so that $L_{0}=-q y$ and $L_{1}=x+(p-q) y$.

We then have the following continued fraction expression for the degree $d$ part $\left(\mathfrak{t}_{\varpi}\right)_{d}$ of the Todd power series of a two-dimensional cone $\varpi$.

Theorem 5. For $\varpi$ a cone of type $(p, q)$ as above, and for $d=2 n+2 \geq 2$ an even integer, we have:

$$
\begin{aligned}
\left(\mathfrak{t}_{\varpi}\right)_{2 n+2}(x, y)= & -q x y \sum_{i=1}^{s} P_{n}\left(L_{i-1}, L_{i}\right)-\lambda_{2 n+2} q x y \sum_{i=1}^{s-1} a_{i} R_{n}\left(L_{i-1}, L_{i+1}\right) \\
& +\lambda_{2 n+2}\left(x L_{1}^{2 n+1}-y L_{s-1}^{2 n+1}\right) .
\end{aligned}
$$

If $d \geq 1$ is odd, then $\left(\mathfrak{t}_{\varpi}\right)_{d}(x, y)=\frac{1}{2} \lambda_{d-1} q^{d-1} x y\left(x^{d-2}+y^{d-2}\right)$.

Proof. It will be convenient to choose coordinates so that $\varpi=\langle(0,-1),(q, q-p)\rangle$ (which is easily seen to be lattice equivalent to the cone $\langle(1,0),(p, q)\rangle$ ). We now subdivide $\varpi$ into nonsingular cones. It is well known that for two-dimensional cones this can be done in a canonical way, and that the resulting subdivision has an explicit expression in terms of continued fractions [Fu, Section 2.6]. In our coordinate system, the rays of this nonsingular subdivision of $\varpi$ are given by

$$
\begin{aligned}
\beta_{0} & =(0,-1), \\
\beta_{1} & =(1,0), \\
\beta_{2} & =\left(a_{1}, 1\right), \\
& \cdots \\
\beta_{s} & =(q, q-p) .
\end{aligned}
$$

Thus we have $\beta_{i+1}+\beta_{i-1}=a_{i} \beta_{i}$, which implies that $\beta_{i}=\left(\gamma_{i}, \delta_{i}\right)$.

The cone $\varpi$ is subdivided into cones $\varpi_{i}=\left\langle\beta_{i-1}, \beta_{i}\right\rangle, i=1, \ldots, s$. The $N$ additivity formula of Proposition 2.7] implies that:

$$
\mathfrak{s}_{\varpi}(x, y)=\sum_{i=1}^{s} \mathfrak{s}_{\varpi_{i}}\left(\varpi_{i}^{-1} \varpi(x, y)^{t}\right),
$$


where again we have identified the 2-dimensional cone $\varpi$ with the 2-by-2 matrix whose columns are the primitive generators of $\varpi$. One easily sees that this becomes

$$
\mathfrak{s}_{\varpi}(x, y)=\sum_{i=1}^{s} \mathfrak{s}_{\varpi_{i}}\left(-L_{i-1}, L_{i}\right)
$$

Rewriting the equation in terms of the $\mathfrak{t}_{\varpi}$ yields

$$
\mathfrak{t}_{\varpi}(x, y)=-q x y \sum_{i=1}^{s} \frac{\mathfrak{t}_{\varpi_{i}}\left(-L_{i-1}, L_{i}\right)}{L_{i-1} L_{i}} .
$$

Since every $\varpi_{i}$ is nonsingular, we have

$$
\mathfrak{t}_{\varpi_{i}}(X, Y)=g(X) g(Y)
$$

where $g(z)=z /\left(1-e^{-z}\right)$. Now consider the degree $d$ part of the above. We will assume $d=2 n+2$ is even with $n>0$, and leave the other (easier) cases to the reader. The degree $d$ part of $g\left(L_{i-1}\right) g\left(L_{i}\right)$ may be written as:

$$
-L_{i-1} L_{i} P_{n}\left(-L_{i-1}, L_{i}\right)+\lambda_{d}\left(L_{i-1}^{d}+L_{i}^{d}\right) .
$$

Summing the first term above yields the first term in the equation of the theorem. So it suffices to examine the remaining term:

$$
-\lambda_{d} q x y \sum_{i=1}^{s} \frac{L_{i-1}^{d}+L_{i}^{d}}{L_{i-1} L_{i}} .
$$

Using the relation $L_{i-1}+L_{i+1}=a_{i} L_{i}$, the sum above may be rewritten as

$$
\sum_{i=1}^{s-1} a_{i} \frac{L_{i-1}^{d-1}+L_{i+1}^{d-1}}{L_{i-1}+L_{i+1}}+\left(\frac{L_{1}^{d-1}}{L_{0}}+\frac{L_{s-1}^{d-1}}{L_{s}}\right) .
$$

Keeping in mind that $L_{0}=-q y$ and $L_{s}=q x$, Theorem 5 follows easily.

Corollary 2.10. For a two-dimensional cone $\sigma=\left\langle\rho_{1}, \rho_{2}\right\rangle$ of multiplicity $q$ we have:

$$
\mathfrak{t}_{\sigma}\left(h_{1}, h_{2}\right)-\frac{1}{2} \mathfrak{t}_{\left\langle\rho_{1}\right\rangle}\left(q h_{1}\right) h_{2}-\frac{1}{2} \mathfrak{t}_{\left\langle\rho_{2}\right\rangle}\left(q h_{2}\right) h_{1}=\mathfrak{t}_{\sigma}^{e v}\left(h_{1}, h_{2}\right)-\frac{q h_{1} h_{2}}{2}
$$

where $\mathfrak{t}_{\sigma}^{e v}$ is the even total degree part of the power series $\mathfrak{t}_{\sigma}$.

Proof. It follows immediately from (and in fact is equivalent to) the formula for the odd part of the Todd power series $\mathfrak{t}_{\sigma}$ given by Theorem 5 .

We now prove Theorem 3 , Let $\sigma$ be a two-dimensional cone of type $(p, q)$ in a lattice $N$, and let $a_{i}, h_{i}, k_{i}$ and $X_{i}$ be as in Theorem 3 The dual cone $\check{\sigma}$ in the dual lattice $M$ is easily seen to have type $(-p, q)$ and so we may choose coordinates in $M$ so that $\check{\sigma}=\langle(0,-1),(q, p)\rangle$ in $\mathbb{Z}^{2}$. Furthermore, the negative-regular continued fraction expansion of $q / p$ corresponds naturally to the desingularization of the dual cone $\check{\sigma}$. Explicitly, the desingularization of $\check{\sigma}$ is given by the subdivision

$$
\begin{aligned}
\rho_{0} & =(0,-1), \\
\rho_{1} & =(1,0), \\
\rho_{2} & =\left(b_{1}, 1\right), \\
& \cdots \\
\rho_{r} & =(q, p) .
\end{aligned}
$$


One has $\rho_{i+1}+\rho_{i-1}=b_{i} \rho_{i}$ and thus $\rho_{i}=\left(h_{i}, k_{i}\right)$. Applying Theorem 5 to $\check{\sigma}$ expresses $\mathfrak{t}_{(-p, q)}$ in terms of the $b_{i}$. However $\mathfrak{t}_{(-p, q)}$ is related to $\mathfrak{t}_{(p, q)}$ via the relation

$$
\mathfrak{t}_{(p, q)}(x, y)=\mathfrak{t}_{(-p, q)}(-x, y)+\frac{q x y}{1-e^{-q y}}
$$

In this way, we obtain an expression for $\mathfrak{t}_{(p, q)}$ in terms of the $b_{i}$, which concludes the proof of Theorem 3 .

2.4. Zeta function values in terms of Todd power series. In this section, we prove Theorem 2 which expresses values of the zeta function of a two-dimensional cone in terms of the Todd power series. Three ingredients are involved in the proof of this theorem: an asymptotic series formula (Lemma2.11), a polytope summation formula (Proposition 2.12) and a cone summation formula (Proposition 2.13). We begin with the first ingredient:

Lemma 2.11 ([ Za44, Proposition 2]). Let $\phi(s)=\sum_{\lambda>0} a_{\lambda} \lambda^{-s}$ be a Dirichlet series where $\{\lambda\}$ is a sequence of positive real numbers converging to infinity. Let $E(t)=$ $\sum_{\lambda>0} a_{\lambda} e^{-\lambda t}$ be the corresponding exponential series. Assume that $E(t)$ has the following asymptotic expansion as $t \rightarrow 0$ :

$$
E(t) \sim \sum_{n=-1}^{\infty} c_{n} t^{n}
$$

Then it follows that

- $\phi(s)$ can be extended to a meromorphic function on $\mathbb{C}$.

- $\phi(s)$ has a simple pole at $s=1$, and no other poles.

- The values of $\phi$ at nonpositive integers are given by: $\phi(-n)=(-1)^{n} n ! c_{n}$.

We now present our second ingredient, a polytope summation formula. This proposition is a variant of the lattice point formula of [BV2]. A polytope of dimension $n$ is called simple if each of its vertices lies on exactly $n$ facets $(n-1$-dimensional faces) of the polytope.

Proposition 2.12. Let $N$ be an $n$-dimensional lattice, $P$ a simple lattice polytope in $M$ and $\Sigma$ its associated fan in $N$. For every analytic function $\phi: M_{\mathbb{R}} \rightarrow \mathbb{R}$, we have the following asymptotic expansion as $t \rightarrow 0$ :

$$
\sum_{a \in P \cap M} \phi(t a) \sim \mathfrak{t}_{\Sigma}\left(\frac{\partial}{\partial h}\right) \diamond \int_{P(h)} \phi(t u) d u
$$

where $\mathfrak{t}_{\Sigma}$ is the Todd power series of [BV2, Definition 10].

Proof. First of all, the meaning of the right hand side is as follows: we consider the degree $k$ Taylor expansion $\phi=\phi_{k}+R_{k}$ of $\phi$, where $\phi_{k}$ is a polynomial in $M_{\mathbb{R}}$ of degree $k$ and $R_{k}$ is the remainder satisfying $\lim _{a \rightarrow 0}|a|^{k} R_{k}(a)=0$. It follows that $\int_{P(h)} \phi_{k}(t u) d u$ is a polynomial in $t$ and $h$ of degree $k$ (with respect to $t$ ) and that $\int_{P(h)} R_{k}(t u) d u=o\left(t^{k}\right)$ at $t=0$ (with the notation that $f(t)=o\left(t^{k}\right)$ if and only if $\left.\lim _{t \rightarrow 0} f(t) t^{-k}=0\right)$. Thus,

$$
\mathfrak{t}_{\Sigma}\left(\frac{\partial}{\partial h}\right) \diamond \int_{P(h)} \phi(t u) d u=\mathfrak{t}_{\Sigma}\left(\frac{\partial}{\partial h}\right) \diamond \int_{P(h)} \phi_{k}(t u) d u+o\left(t^{k}\right) .
$$


On the other hand,

$$
\begin{aligned}
\sum_{a \in P \cap M} \phi(t a) & =\sum_{a \in P \cap M} \phi_{k}(t a)+\sum_{a \in P \cap M} R_{k}(t a) \\
& =\sum_{a \in P \cap M} \phi_{k}(t a)+o\left(t^{k}\right) .
\end{aligned}
$$

Brion and Vergne [BV2, Theorem 11] prove that for every polynomial function (such as $\phi_{k}$ ) on $M_{\mathbb{R}}$ we have:

$$
\sum_{a \in P \cap M} \phi_{k}(t a)=\mathfrak{t}_{\Sigma}\left(\frac{\partial}{\partial h}\right) \diamond \int_{P(h)} \phi_{k}(t u) d u
$$

which concludes the proof.

We call a function $\phi: \mathbb{R}^{n} \rightarrow \mathbb{R}$ rapidly decreasing if it is analytic and, for every constant coefficients differential operator $D$ and every subset $I$ of $[n]=\{1, \ldots, n\}$, the restriction $\left.D(\phi)\right|_{I}$ obtained by setting $x_{i}=0$ for $i \notin I$ is in $L^{1}\left(\mathbb{R}_{+}^{I}\right)$. Examples of rapidly decreasing functions can be obtained by setting $\phi=P \exp (Q)$ where $P$ is a polynomial on $\mathbb{R}^{n}$ and $Q: \mathbb{R}^{n} \rightarrow \mathbb{R}$ is totally positive, i.e., its restriction to $\mathbb{R}_{+}^{I}$ takes positive values for every subset $I$ of $[n]$.

Proposition 2.13. Let $N$ be an n-dimensional lattice. For every $\sigma \in \mathcal{C}^{n}(N)$ and every rapidly decreasing analytic function $\phi: M_{\mathbb{R}} \rightarrow \mathbb{R}$, we have the following asymptotic expansion as $t \rightarrow 0$ :

$$
\sum_{a \in \tilde{\sigma} \cap M} \phi(t a) \sim \mathfrak{t}_{\sigma}\left(\frac{\partial}{\partial h}\right) \diamond \int_{\check{\sigma}(h)} \phi(t u) d u .
$$

Proof. First of all, the right hand side of the above equation has the following meaning: consider the decomposition $\mathfrak{t}_{\sigma}=\sum_{k} \mathfrak{t}_{\sigma, k}$ of the power series $\mathfrak{t}_{\sigma}$, where $\mathfrak{t}_{\sigma, k}$ is a homogeneous polynomial of degree $k$. A change of variables $v=t u$ implies that

$$
\begin{aligned}
\mathfrak{t}_{\sigma, k}\left(\frac{\partial}{\partial h}\right) \diamond \int_{\check{\sigma}(h)} \phi(t u) d u & =\mathfrak{t}_{\sigma, k}\left(\frac{\partial}{\partial h}\right) \diamond \int_{\check{\sigma}(t h)} \phi(v) d v / t^{n} \\
& =t^{k-n} \mathfrak{t}_{\sigma, k}\left(\frac{\partial}{\partial h}\right) \diamond \int_{\check{\sigma}(h)} \phi(v) d v
\end{aligned}
$$

is a multiple of $t^{k-n}$, thus the right hand side is defined to be the Laurent power series in $t$ given by

$$
\sum_{k=0}^{\infty} t^{k-n} \mathfrak{t}_{\sigma, k}\left(\frac{\partial}{\partial h}\right) \diamond \int_{\check{\sigma}(h)} \phi(v) d v .
$$

For the proof of the proposition, truncate in some way the cone $\check{\sigma}$ in $M$ to obtain a simple convex polytope $P$, with associated fan $\Sigma$. Since $\bigcup_{r>0} r P=\check{\sigma}$, using the 
convergence properties of $\phi$, we obtain as $r \rightarrow \infty$ :

$$
\begin{aligned}
\sum_{a \in \check{\sigma} \cap M} \phi(t a) & =\lim _{r} \sum_{a \in r P \cap M} \phi(t a) \\
& \sim \lim _{r} \mathfrak{t}_{\Sigma}\left(\frac{\partial}{\partial h}\right) \diamond \int_{(r P)(h)} \phi(t u) d u \\
& =\lim _{r} \mathfrak{t}_{\Sigma}\left(\frac{\partial}{\partial h}\right) \diamond \int_{(r t P)(t h)} \phi(v) d v / t^{n} \\
& =\lim _{r} \sum_{k} \mathfrak{t}_{\Sigma, k} t^{k-n}\left(\frac{\partial}{\partial h}\right) \diamond \int_{(r t P)(h)} \phi(v) d v \\
& =\sum_{k} \lim _{r} \mathfrak{t}_{\Sigma, k} t^{k-n}\left(\frac{\partial}{\partial h}\right) \diamond \int_{(r t P)(h)} \phi(v) d v \\
& =\sum_{k} \mathfrak{t}_{\sigma, k} t^{k-n}\left(\frac{\partial}{\partial h}\right) \diamond \int_{P(h)} \phi(v) d v,
\end{aligned}
$$

which concludes the proof.

In the case of a two-dimensional lattice $N$ and a rapidly decreasing function $\phi: M_{\mathbb{R}} \rightarrow \mathbb{R}$, using the weight function $w t$ of equation (2) and inclusion-exclusion, we obtain the following

Corollary 2.14. For a two-dimensional cone $\sigma=\left\langle\rho_{1}, \rho_{2}\right\rangle$ of multiplicity $q$ in $N$, we have the asymptotic expansion as $t \rightarrow 0$ :

$$
\sum_{a \in \check{\sigma} \cap M} w t(\check{\sigma}, a) \phi(t a) \sim\left\{\mathfrak{t}_{\sigma}^{e v}\left(\frac{\partial}{\partial h_{1}}, \frac{\partial}{\partial h_{2}}\right)-\frac{q}{2} \frac{\partial^{2}}{\partial h_{1} \partial h_{2}}\right\} \diamond \int_{\check{\sigma}(h)} \phi(t u) d u,
$$

where the right hand side lies in the formal power series ring $t^{-2} \mathbb{R} \llbracket t^{2} \rrbracket$.

Proof of Theorem 2. Recall that $\tau$ is a cone in $M, \sigma$ is its dual in $N$ and $Q$ is homogeneous quadratic, totally positive on $\tau$; thus $e^{-Q}$ is rapidly decreasing on $M_{\mathbb{R}}$. Corollary 2.14 implies that the generating function

$$
Z_{Q, \tau}(t)=\sum_{a \in \tau \cap M} w t(\tau, a) e^{-t Q(a)}=\sum_{a \in \tau \cap M} w t(\tau, a) e^{-Q\left(t^{1 / 2} a\right)}
$$

satisfies the hypothesis of Lemma 2.11, which in turn yields Theorem 2 .

Remark 2.15. Notice that the above proof of Theorem 2 used crucially the fact that $B_{1}=-\frac{1}{2}$ and the definition of the weight function $w t$. If we had weighted the sum defining the zeta function in any other way, the resulting variation of Theorem 2 would not hold.

\section{Proof of Theorem 1}

3.1. Some lemmas. The proof of Theorem 1 will use some lemmas concerning the admissible triples $(M, Q, \tau)$ constructed given a sequence $b=\left(b_{0}, \ldots, b_{r-1}\right)$ which we fix for the rest of this section.

The recursion relation (3) implies that

$$
A_{k}=-p_{k} A_{-1}+q_{k} A_{0} \quad \text { where } \frac{p_{k}}{q_{k}}=\left[b_{0}, \ldots, b_{k-1}\right] \text {. }
$$


Let $p, p^{\prime}, q$ be defined by

$$
\frac{q}{p}=\left[b_{1}, \ldots, b_{r-1}\right] \quad \text { and } \quad p^{\prime}=\text { numerator }\left[b_{1}, \ldots, b_{r-2}\right],
$$

with the understanding that $q=1, p=0, p^{\prime}=0$ if $r=1$ and $p^{\prime}=1$ if $r=2$. It is easy to see that $q, p, p^{\prime}$ are (for fixed $r$ ) polynomials in the $b_{i}$ and that $p p^{\prime}=1 \bmod q$.

Lemma 3.1. The cone $\tau=\left\langle A_{0}, A_{r}\right\rangle$ in $M$ is of type $(-p, q)$ and the dual cone $\sigma$ in $N$ is of type $(p, q)$ where $0 \leq p<q$ as in equation (22) above.

Proof. The cone $\left\langle A_{r}, A_{0}\right\rangle$ is canonically subdivided into nonsingular cones $\left\langle A_{i+1}, A_{i}\right\rangle$ (for $i=0, \ldots, r-1$ ), and using equation (3), it follows that $\tau$ is of type $\left(c_{1}, q\right)$ where $0 \leq c_{1}<q$ and $\frac{q}{q-c_{1}}=\left[b_{1}, \ldots, b_{r-1}\right]$. Therefore the dual cone $\sigma$ in $N$ is of type $(p, q)$ where $p=q-c_{1}$. Thus $(p, q)$ satisfies equation (22).

Lemma 3.2. Let $l, m \in \mathbb{Z}$ with $l \neq m$. Then the coefficients of $x^{2}, x y$ and $y^{2}$ of the quadratic form $Q\left(x A_{l}+y A_{m}\right.$ ) are given (for fixed $r$ ) by polynomials in $b_{i}$ with integer coefficients. Moreover,

$$
\begin{aligned}
Q\left(x A_{-1}+y A_{1}\right)= & \left(q p^{\prime} b_{0}+1-p p^{\prime}\right) x^{2}+\left(q b_{0} \theta+2\left(p p^{\prime}-1\right)\right) x y \\
& +\left(q p b_{0}+1-p p^{\prime}\right) y^{2} \\
Q\left(x A_{r}+y A_{0}\right)= & q\left(x^{2}+\theta x y+y^{2}\right)
\end{aligned}
$$

where $\theta=b_{0} q-p-p^{\prime}$.

Proof. The first part follows immediately from equation (21) and from the fact that $Q\left(x A_{-1}+y A_{0}\right)=C x^{2}-B x y+A y^{2}$, where $A, B, C$ are polynomials in the $b_{i}$ with integer coefficients; see Section 1.1. In fact, the $A, B, C$ can be calculated (using their definition) in terms of the $b_{i}$ as follows:

$$
A=q, \quad B=-b_{0} q+p-p^{\prime}, \quad \text { and } \quad C=q_{r-1}=b_{0} p^{\prime}+\left(1-p p^{\prime}\right) / q .
$$

This, together with a change of variables formula from $\left\{A_{-1}, A_{0}\right\}$ to $\left\{A_{r}, A_{0}\right\}$ and to $\left\{A_{-1}, A_{1}\right\}$ given by equation (21), implies the other assertions of the Lemma.

\subsection{Proof of Theorem 1.}

Proof. The main idea is to use Theorem 2 which calculates the zeta values in terms of the Todd operator of $\sigma$, and Theorem 3 which expresses the Todd operator in terms of the $b_{i}$. The expression that we obtain for the zeta values differ from the one of equation (5) by an error term, which vanishes identically, as one can show by an explicit calculation.

Now, for the details, we begin by calculating the integral $\int_{\tau\left(h_{1}, h_{2}\right)} e^{-Q(u)} d u$. Using the parametrization $\mathbb{R}^{2} \rightarrow M_{\mathbb{R}}$ given by $\left(u_{1}, u_{2}\right) \rightarrow u_{1} A_{r}+u_{2} A_{0}$, it follows that the preimage of $\tau(x, y)$ in $\mathbb{R}^{2}$ is given by $\left\{\left(u_{1}, u_{2}\right) \mid u_{1} \geq-x / q, u_{2} \geq-y / q\right\}$. Thus we have:

$$
\int_{\tau(x, y)} e^{-Q(u)} d u=q \int_{u_{1}=-x / q}^{\infty} \int_{u_{2}=-y / q}^{\infty} e^{-Q\left(u_{1} A_{r}+u_{2} A_{0}\right)} d u_{2} d u_{1} .
$$

Differentiating, we get

$$
\left(\frac{\partial}{\partial x}\right)\left(\frac{\partial}{\partial y}\right) \diamond \int_{\tau(x, y)} e^{-Q(u)} d u=\frac{1}{q} e^{-Q\left(-x A_{r} / q-y A_{0} / q\right)}=\frac{1}{q} e^{-Q\left(x A_{r} / q+y A_{0} / q\right)} .
$$


Using the notation of Theorem $[3$ and the elementary identity

$$
\begin{gathered}
\left.\left(\alpha \frac{\partial}{\partial \bar{x}}+\beta \frac{\partial}{\partial \bar{y}}\right)^{i}\left(\gamma \frac{\partial}{\partial \bar{x}}+\delta \frac{\partial}{\partial \bar{y}}\right)^{j}\right|_{\substack{\bar{x}=\alpha a+\gamma b \\
\bar{y}=\beta a+\delta b}} f(\bar{x}, \bar{y}) \\
=\left.\left(\frac{\partial}{\partial x}\right)^{i}\left(\frac{\partial}{\partial y}\right)^{j}\right|_{\substack{x=a \\
y=b}} f(\alpha x+\gamma y, \beta x+\delta y)
\end{gathered}
$$

(and temporarily abbreviating $\frac{\partial}{\partial x}$ by $x$ ), we obtain that

$$
\begin{gathered}
\left(q x y X_{l}^{a} X_{m}^{b}\right) \diamond \int_{\tau(x, y)} e^{-Q(u)} d u=X_{l}^{a} X_{m}^{b} \diamond e^{-Q\left(x A_{r} / q+y A_{0} / q\right)} \\
=\left(\frac{\partial}{\partial x}\right)^{a}\left(\frac{\partial}{\partial y}\right)^{b} \diamond e^{-Q\left(x A_{l}+y A_{m}\right)}
\end{gathered}
$$

as well as

$$
\begin{aligned}
& \left(x X_{1}^{2 n+1}+y X_{r-1}^{2 n+1}\right) \diamond \int_{\tau(x, y)} e^{-Q(u)} d u \\
& =\left.\frac{1}{q^{n+1}} \int_{0}^{\infty}\left(\left(\frac{\partial}{\partial x}+p \frac{\partial}{\partial y}\right)^{2 n+1}+\left(\frac{\partial}{\partial x}+p^{\prime} \frac{\partial}{\partial y}\right)^{2 n+1}\right)\right|_{x=0} e^{-\left(x^{2}+\theta x y+y^{2}\right)} d y .
\end{aligned}
$$

The above, together with Theorem 3, implies that:

$$
\begin{aligned}
\zeta_{Q, \tau}(-n)= & (-1)^{n} n !\left\{\sum_{i=0}^{r-1} P_{n}\left(\frac{\partial}{\partial x}, \frac{\partial}{\partial y}\right) \diamond e^{-Q\left(x A_{i-1}+y A_{i}\right)}\right. \\
& \left.+\lambda_{2 n+2} \sum_{i=0}^{r-1} b_{i} R_{n}\left(\frac{\partial}{\partial x}, \frac{\partial}{\partial y}\right) \diamond e^{-Q\left(x A_{i-1}+y A_{i+1}\right)}+E_{2 n+2}(b)\right\}
\end{aligned}
$$

where

$$
\begin{aligned}
& E_{2 n+2}(b)= \lambda_{2 n+2}\left\{-b_{0} R_{n}\left(\frac{\partial}{\partial x}, \frac{\partial}{\partial y}\right) e^{-Q\left(x A_{-1}+y A_{1}\right)}\right. \\
&-\left.\frac{1}{q^{n+1}} \int_{0}^{\infty}\left(\left(\frac{\partial}{\partial x}+p \frac{\partial}{\partial y}\right)^{2 n+1}+\left(\frac{\partial}{\partial x}+p^{\prime} \frac{\partial}{\partial y}\right)^{2 n+1}\right)\right|_{x=0} \\
&\left.\times e^{-\left(x^{2}+\theta x y+y^{2}\right)} d x\right\} .
\end{aligned}
$$

Fixing $r$, the length of the sequence $\left(b_{0}, \ldots, b_{r-1}\right)$, Lemmas 3.2 and 3.3 (below) can be used to express $E_{2 n+2}(b)$ as a polynomial in $1 / q, p, p^{\prime}, b_{0}$. An explicit but lengthy calculation implies that $E_{2 n+2}(b)=0$ for any $b$. Since $Q$ is homogeneous quadratic and $P_{n}$ homogeneous of degree $2 n$, it follows that

$$
P_{n}\left(\frac{\partial}{\partial x}, \frac{\partial}{\partial y}\right) \diamond e^{-Q\left(x A_{i-1}+y A_{i}\right)}=\frac{(-1)^{n}}{n !} P_{n}\left(\frac{\partial}{\partial x}, \frac{\partial}{\partial y}\right)\left(Q\left(x A_{i-1}+y A_{i}\right)^{n}\right)
$$

which concludes the proof of Theorem 1 . The value of $\zeta_{Q, \tau}(0)$ follows easily using $\lambda_{1}=1 / 2, \lambda_{2}=1 / 12$. 
Lemma 3.3 ([पa4] $)$. For every $i, j \geq 0$ with $i+j$ even, we have:

$$
\begin{aligned}
& \left(\frac{\partial}{\partial x}\right)^{i}\left(\frac{\partial}{\partial y}\right)^{j} \diamond e^{-\left(a x^{2}+b x y+c y^{2}\right)} \\
& \quad=i ! j !(-1)^{(i+j) / 2} a^{i / 2} c^{j / 2} \sum_{i_{2}=0 ; i_{2} \equiv i \bmod 2}^{\min \{i, j\}} \frac{\left(a^{-1 / 2} b c^{-1 / 2}\right)^{i_{2}}}{\left(\left(i-i_{2}\right) / 2\right) ! i_{2} !\left(\left(j-i_{2}\right) / 2\right) !} .
\end{aligned}
$$

Furthermore, for every $n \geq 1$ we have:

$$
\begin{aligned}
\int_{y=0}^{\infty} & \left(\frac{\partial}{\partial x}\right)^{2 n-1} \diamond e^{-\left(a x^{2}+b x y+c y^{2}\right)} d y \\
& =-\frac{(2 n-1) !}{2 c^{n}} \sum_{r=0}^{n-1}(-1)^{r} \frac{(n-1-r) !}{r !(2 n-1-2 r) !} a^{r} b^{2 n-1-2 r} c^{r}
\end{aligned}
$$

\section{Dedekind SUmS IN TERMS OF TODd POWER SERIES}

In this section we give a proof of Theorem 4. To do this we will use Proposition 2.3, which can be used to express the coefficients $f_{i, j}$ as a sum of rational numbers, which turn out to equal products of certain values of the Benoulli polynomials. (Note, in contrast, that Proposition 2.4 expresses this same number in terms of roots of unity instead of rational numbers.)

Let $p$ and $q$ be as in the statement of Theorem 4 and let $\sigma$ be the cone $\langle(1,0),(p, q)\rangle$ in $\mathbb{Z}^{2}$. We let $\rho_{1}=(1,0)$ and $\rho_{2}=(p, q)$ denote the generators of this cone. The left hand side of Theorem $4, f_{i, j}(p, q)$, equals the coefficient of $x^{i} y^{j}$ in the power series $\mathfrak{t}_{\sigma}(x, y)$. We now compute this power series using Proposition 2.3. This proposition contains an expansion for the power series $\mathfrak{s}_{\sigma}$, which is equivalent to the following expansion of $\mathfrak{t}_{\sigma}$ :

$$
\mathfrak{t}_{\sigma}(x, y)=q x y \sum_{m \in \check{\sigma} \cap M} e^{-\left(\left\langle m, \rho_{1}\right\rangle x+\left\langle m, \rho_{2}\right\rangle y\right)} .
$$

Every point of $\check{\sigma} \cap M$ can be written uniquely as a nonnegative integral combination of the generators $u_{1}=(q,-p)$ and $u_{2}=(0,1)$ of $\check{\sigma}$, plus a lattice point in the semiopen parallelepiped

$$
P=\left\{c u_{1}+d u_{2} \mid c, d \in[0,1)\right\} .
$$

Using $\left\langle u_{1}, \rho_{1}\right\rangle=\left\langle u_{2}, \rho_{2}\right\rangle=q$, it follows that

$$
\mathfrak{t}_{\sigma}(x, y)=q x y \frac{1}{1-e^{-q x}} \frac{1}{1-e^{-q y}} \sum_{m \in P \cap M} e^{-\left(\left\langle m, \rho_{1}\right\rangle x+\left\langle m, \rho_{2}\right\rangle y\right)} .
$$

One finds also that

$$
P=\left\{\left(k,\left\{\frac{p k}{q}\right\}-\frac{p k}{q}\right): k=0, \ldots, q-1\right\},
$$

where $\{x\} \in[0,1)$ denotes the fractional part of $x$. (Note this is slightly different from $\langle x\rangle \in(0,1]$, which appears in the definition of $s_{i, j}$ : by definition, $\langle 0\rangle=1$, while $\{0\}=0$.) One then finds that

$$
\mathfrak{t}_{\sigma}(x, y)=q x y \frac{1}{1-e^{-q x}} \frac{1}{1-e^{-q y}} \sum_{k=0}^{q-1} e^{-q\left(\frac{k}{q} x+\left\{\frac{k p}{q}\right\} y\right)} .
$$


We may then compute $f_{i, j}(p, q)$ as the coefficient of $x^{i} y^{j}$ in the above expression. It is convenient to replace $x$ and $y$ with $-x$ and $-y$, which introduces a factor of $(-1)^{i+j}$. We obtain

$$
f_{i, j}(p, q)=(-1)^{i+j} q^{i+j-1} \sum_{k=0}^{q-1} \operatorname{coeff}\left(x^{i} ; \frac{x e^{\frac{k}{q} x}}{1-e^{x}}\right) \operatorname{coeff}\left(y^{i} ; \frac{y e^{\left\{\frac{k p}{q}\right\} y}}{1-e^{y}}\right) .
$$

It is then clear that we can write the above sum in terms of values of the Bernoulli polynomials, as follows:

$$
f_{i, j}(p, q)=(-1)^{i+j} q^{i+j-1} \sum_{k=0}^{q-1} \operatorname{Ber}_{i}\left(\frac{k}{q}\right) \operatorname{Ber}_{j}\left(\left\{\frac{k p}{q}\right\}\right)
$$

Using the identity

$$
\operatorname{Ber}_{j}(\lambda)=(-1)^{j} \operatorname{Ber}_{j}(1-\lambda),
$$

we may rewrite our expression as

$$
f_{i, j}(p, q)=(-1)^{i} q^{i+j-1} \sum_{k=0}^{q-1} \operatorname{Ber}_{i}\left(\frac{k}{q}\right) \operatorname{Ber}_{j}\left(\left\langle-\frac{k p}{q}\right\rangle\right) .
$$

The sum on the right hand side is easily seen to equal the sum defining $s_{i, j}(p, q)$, except for a possible discrepancy in the $k=0$ term. It follows easily from the definitions that the $k=0$ terms actually match unless $i=j=1$, or $i=1$ and $j$ is even, or $j=1$ and $i$ is even. In these cases, we need the correction terms which appear in the statement of the Theorem.

\section{REFERENCES}

[AV] T. Apostol, T. Vu, Identities for sums of Dedekind type, J. Number Theory 14 (1982) 391-396. MR 83h:10054

[Ba] A. I. Barvinok, A polynomial time algorithm for counting integral points in polyhedra when the dimension is fixed, Math. Oper. Res. 19 (1994) no.4 769-779. MR 96c:52026

[BP] A. I. Barvinok and J. E. Pommersheim, An algorithmic theory of lattice points in polyhedra, New Perspectives in Mathematics, MSRI, 1999. CMP 2000:07

[Br] M. Brion, Points entier dans les polyèdres convexes, Ann. Scient. Éc. Norm. Sup. 21 (1988) no.4 653-663. MR 90d:52020

[BV1] M. Brion and M. Vergne, An equivariant Riemann-Roch theorem for simplicial toric varieties, J. Reine Angew. Math 482 (1997) 67-92. MR 98a:14067

[BV2] Lattice points in simple polytopes, Journal of AMS 10 (1997) 371-392. MR 98a:11132

[CS] S. Cappell, J. Shaneson, Genera of algebraic varieties and counting lattice points, Bull. Amer. Math. Soc. 30 (1994) 62-69. MR 94f:14018

[CN1] P. Cassou-Noguès, Valeurs aux entiers négatifs des fonctions zêta et fonctions zêta p-adiques, Inventiones Math. 51 (1979) 29-59. MR 80h:12009b

[CN2] Valeurs aux entiers négatifs des séries de Dirichlet associées a un polynôme I, Journal of Number Theory 14 (1982) 32-64. MR 83e:12012]

[CN3] Valeurs aux entiers négatifs des séries de Dirichlet associées a un polynôme II, III, Amer. J. Math 106 (1984) 255-299 and 109 (1987) 71-89. MR 85b:11109. MR 88b:11056

[Da] V. I. Danilov, The geometry of toric varieties, Russian Math. Surveys 33 (1978) 97-154. MR 80g:14001

[DR] R. Diaz, S. Robins, The Ehrhart polynomial of a lattice polytope, Annals of Math. 145 (1997) 503-518. MR 98e:11117a MR 98e:11117b

[Fu] W. Fulton, Introduction to toric varieties, Annals of Math. Studies 131, Princeton, New Jersey, 1993. MR 94g:14028 
[Gu] V. Guillemin, Riemann-Roch for toric orbifolds, J. Diff. Geom. 45 (1997) 53-73. MR 98a:58075

[Ha] U. Halbritter, Some new reciprocity formulas for generalized Dedekind sums, Results Math. 8 (1985) 21-46. MR 87a:11043

[Hs] D. Hayes, Brumer elements over a real quadratic base field, Exposition. Math. 8 no.2 (1990) 137-184. MR 92a:11142

[KK] J.M. Kantor, A.G. Khovanskii, Integral points in convex polyhedra, combinatorial Riemann-Roch and generalized MacLaurin formulae, IHES (1992) 932-937.

[KP] A.G. Khovanskii, A.V. Pukhlikov, A Riemann-Roch theorem for integrals and sums of quasipolynomials over virtual polytopes, St. Petersburg Math. J. 4 (1993) 789-912. MR 94c:14044

[Kl] H. Klingen, Über die Werke der Dedekindsche Zeta Funktion, Math. Ann. 145 (1962) $265-272$.

[Li] S. Lichtenbaum, Talk at I.A.S., mimeographed notes 1971.

[Me] C. Meyer, Über die Berechnung der Klassenzahl abelscher Körper quadratischen Zahlkörpern, Akademie-Verlag Berlin, 1955. MR 19:531f

[Mo] R. Morelli, Pick's Theorem and the Todd class of a toric variety, Adv. Math. 100:2 (1993) 183-231. MR 94j:14048

[P1] J. E. Pommersheim, Toric varieties, lattice points and Dedekind sums, Math. Ann. 295 (1993) 1-24. MR 94c:14043

[P2] $\quad$, Products of cycles and the Todd class of a toric variety, Journal of AMS 9 (1996) no. 3, 813-826. MR 96j:14037

[P3] , Barvinok's algorithm and the Todd class of a toric variety, J. Pure Appl. Alg. 117 \& 118 (1997) 519-533. MR 98g:14067

[RG] H. Rademacher, E. Grosswald, Dedekind sums, Carus Math. Monographs, 16 Mathematical Association of America, Washington D.C., 1972. MR 50:9767

[Sc1] R. Sczech, Eisenstein cocycles for $G L_{2}(\mathbb{Q})$ and values of L-functions in real quadratic fields, Comment. Math. Helvetici 67 (1992) 363-382. MR 93h:11047

[Sc2] Eisenstein group cocycles for $G L_{n}(\mathbb{Q})$ and values of $L$-functions, Inventiones 113 (1993) 581-616. MR 94j:11049

[Sh1] T. Shintani, On evaluation of zeta functions of totally real algebraic number fields at nonpositive integers, J. Fac. Sci. Univ. Tokyo, Sec. 1A, 23 (1976) 393-417. MR 55:266

[Sh2] - On special values of zeta functions of totally real algebraic number fields, Proc. Intern. Congress of Mathematicians, Helsinki (1978) 591-597. MR 81e:12016

[Si1] C. L. Siegel, Bernoulische Polynome und Quadratische Zahlkörper, Nachr. Akad. Wiss. Göttingen Math. Physik. 3 (1970) 7-38. MR 38:2123

[Si2] U Über die Fourierschen Koeffizienten von Modulformen, Nachr. Akad. Wiss. Göttingen Math. Physik. 3 (1970) 15-56. MR 44:2706

[So] D. Solomon, Algebraic properties of Shintani's generating functions: Dedekind sums and cocycles on $P G L_{2}(\mathbb{Q})$, Compositio Math. 112 (1998) 333-362. MR 99g:11137

[St] G. Stevens, The Eisenstein measure and real quadratic fields, in the proceedings of the International Number Theory Conference (Université Laval, 1987), J.M. de Koninck and C. Levesque eds., de Gruyter, 1989. MR 90m:11077

[Za1] D. Zagier, Higher dimensional Dedekind sums, Math. Ann. 202 (1973) 149-172. MR 50:9801

[Za2] , A Kronecker limit formula for real quadratic fields, Math. Ann. 213 (1975) 153-182. MR 51:3123

[Za3] - Nombres de classes et fractions continues, Soc. Math. France Asterisque 24-25 (1975) 81-97. MR 51:10293

[Za4] Valeurs des fonctions zêta des corps quadratiques réels aux entiers négatifs, Soc. Math. France Asterisque 41-42 (1977) 135-151. MR 56:316

School of Mathematics, Georgia Institute of Technology, Atlanta, Georgia 303320160

E-mail address: stavros@math.gatech.edu

Department of Mathematics, Pomona College, 610 North College Ave., Claremont, California 91711

E-mail address: jpommersheim@pomona.edu 